\title{
DESARROLLO Y CAMBIO CLIMÁTICO
}

\author{
Miguel Ángel ElizAlde CARRANZA \\ Profesor de Derecho Internacional Público \\ Universitat Pompeu Fabra \\ miguelangel.elizalde@upf.edu
}

Recibido: 19 de abril de 2010 / Aceptado: 21 de mayo de 2010

RESUMEN: El cambio climático constituye una de las principales amenazas para el desarrollo económico mundial, especialmente de los países más pobres. A su vez, en el ámbito del desarrollo, se encuentran los principales retos que la comunidad internacional debe resolver para el establecimiento de un nuevo régimen internacional del cambio climático. Esta nota explora la relación entre ambas cuestiones.

RESUM: El canvi climàtic constitueix una de les amenaces principals per al desenvolupament econòmic mundial, especialment dels països més pobres. A la vegada, en l'àmbit del desenvolupament, es troben els principals reptes que la comunitat internacional ha de resoldre per a l'establiment d'un nou règim internacional del canvi climàtic. Aquesta nota explora la relació entre ambdues qüestions.

\begin{abstract}
Climate change is one of the most important menaces for the World economic development, specially in the case of the poorest countries. At the same time, in the domain of the development we can find the main challenges for the international community, in order to establish a new international regime on climate change. This note tries to explore the relationship between these issues.
\end{abstract}

PALABRAS CLAVE: Cambio climático — desarrollo 
PARAULES CLAU: Canvi climàtic — desenvolupament

KEYWORDS: Climate change — development

Sumario: I. Introducción. II. El cambio climático. 1. El umbral de cambio climático considerado peligroso. 2. La estabilización de emisiones de GEI: Mitigación. III. El cambio climático y sus vínculos con el desarrollo. 1. Los efectos del cambio climático en el desarrollo. 1.1. El declive en la producción agrícola y la seguridad alimentaria. 1.2. La disponibilidad de agua. 1.3. Los efectos en el medio ambiente. 1.4 Los efectos en la salud humana. 1.5 Los efectos en la estabilidad social. 2. La dimensión Norte-Sur. 2.1 La diferencia en la vulnerabilidad frente al cambio climático. 2.2 La responsabilidad común pero diferenciada. 2.3 El imperativo de la adaptación. IV. El camino hacia el nuevo régimen del cambio climático. 1. El régimen internacional en vigor. 2. Los avances en las negociaciones de un régimen postKioto. 3. Los principales retos a superar en el camino hacia un régimen post-Kioto. 3.1. La cooperación multilateral efectiva. 3.2 El financiamiento. 3.3. La transferencia de tecnología. V. Conclusión. VI. Bibliografía.

"El cambio climático es el mayor desafío que ha enfrentado el género humano"

$P N U D^{1}$

\section{INTRODUCCIÓN}

El cambio climático empezó a llamar la atención pública a principios de la década de los 90, tras la publicación del primer informe del Panel Intergubernamental sobre Cambio Climático (IPCC por sus singlas en inglés), máxima autoridad de expertos en la materia, y se veía como un problema ambiental. Poca atención recibían los efectos socioeconómicos asociados ${ }^{2}$. Veinte años después, la visión ha cambiado. El calentamiento global es reconocido hoy por hoy como una de las principales amenazas para el desarrollo mundial. Así lo demuestra el hecho de que, en septiembre de 2009, en la sesión de apertura de la denominada Cumbre sobre el Cambio Climático, el Secretario General de las Naciones Unidas, Ban Ki-moon, calificara al cambio climático como el principal reto geopolítico y económico del siglo 21 por su potencial de afectar el desarrollo, la paz y la prosperidad de la humanidad ${ }^{3}$.

\footnotetext{
1 PNUD, Informe sobre Desarrollo Humano 2007-2008: La lucha contra el cambio climático: Solidaridad frente a un mundo dividido, PNUD, Nueva York, 2007, p. 6.

${ }^{2}$ HUQ, S., REID, H., MURRAY, L.A., Climate change and development links, International Institute for Environment and Development, Londres, 2006, p. 3.

${ }^{3}$ UN News Centre (2009, 22 de septiembre), "Opening remarks to the United Nations Climate Change Summit Plenary". Recuperado el 12 de enero de 2010 de: http://www.un.org/apps/news/infocus/sgspeeches/statments full.asp?statID=582
} 
Las siguientes líneas contienen una breve introducción de algunos de los aspectos más importantes de la relación que existe entre el cambio climático y el desarrollo.

\section{EL CAMBIO CLIMÁTICO}

Las variaciones climáticas son parte del funcionamiento regular de los ecosistemas. En distintos grados, los cambios en el sistema de clima históricamente han sido una constante. Por otro lado, se ha comprobado que el clima de la Tierra también está sufriendo variaciones como consecuencia de las actividades humanas. Los estudios del IPCC han establecido una relación entre las actividades económicas que han tenido lugar desde la revolución industrial hasta nuestros días con un incremento en la temperatura promedio de la Tierra de $0,7^{\circ} \mathrm{C}$ en comparación con la era preindustrial ${ }^{4}$.

Para evitar confusiones, conviene aclarar que en esta nota las referencias al cambio climático se deben entender como las variaciones del clima provocadas directa o indirectamente por el hombre ${ }^{5}$.

El cambio climático de origen humano o antropogénico se debe a que las actividades económicas producen emisiones de diversos gases que se acumulan en la atmósfera por periodos muy largos de tiempo — por eso algunas veces se les llama gases de larga permanencia-. Estos gases capturan parte de la energía solar que en otras circunstancias sería devuelta al espacio como consecuencia del reflejo que genera el contacto de la luz solar con la superficie de la Tierra. A este fenómeno se le conoce comúnmente como "el efecto invernadero" ya que incrementa la temperatura promedio terrestre ${ }^{6}$.

\footnotetext{
${ }^{4}$ IPCC, Cambio climático 2007: Informe de sintesis. Contribución de los Grupos de trabajo I, II y III al Cuarto Informe de evaluación del Grupo Intergubernamental de Expertos sobre el Cambio Climático [Equipo de redacción principal: Pachauri R.K., Reisinger, A. (directores de la publicación)], IPCC, Ginebra, 2007, p. 2.

${ }^{5}$ Esta definición sigue de cerca a la Convención Marco de las Naciones Unidas sobre el cambio climático (CMNUCC) cuyo artículo 1.2 establece que se entiende por "cambio climático" los cambios en el clima que sean provocados directa o indirectamente por las actividades humanas que altera la composición de la atmósfera mundial y que se suma a la variabilidad natural del clima observada durante períodos de tiempo comparables. Véase Convención Marco de las Naciones Unidas sobre el cambio climático, hecha en Nueva York el 9 de mayo de 1992 (BOE, 01 de febrero de 1994, No. 27).

${ }^{6}$ Esto responde a las similitudes que existen con el funcionamiento de los invernaderos en los que, en lugar de gases, son los cristales utilizados en sus estructuras los que capturan energía solar para elevar la temperatura en su interior.
} 
Aunque las actividades humanas generan emisiones de distintos gases de larga permanencia de efecto invernadero - en lo sucesivo GEI -, como el metano $\left(\mathrm{CH}_{4}\right)$, óxido nitroso $\left(\mathrm{N}_{2} \mathrm{O}\right)$ y los halocarbonos, el más importante en términos cuantitativos y por su potencial para retener calor es el $\mathrm{CO}^{2}$. En su informe más reciente, el IPCC ha expuesto que las emisiones mundiales de GEI por efecto de las actividades humanas han aumentado, desde la era preindustrial, en un $70 \%$ entre 1970 y 2004, siendo el CO2 la fuente principal con un aumento de emisiones del $80 \%{ }^{7}$ Por sectores, las fuentes más importantes son el suministro de energía (26\%), el transporte (13\%), la industria (19\%), la vivienda y el comercio (8\%), la silvicultura (incluye la deforestación, 17\%) y la agricultura $(14 \%){ }^{8}$ Más aún, el IPCC sostiene que con las políticas actuales, las emisiones mundiales de GEI seguirán aumentando en los próximos decenios y podrían traducirse, a partir del 2020 , en un incremento de $1^{\circ} \mathrm{C}$ cada 10 años 9 .

\section{El umbral de cambio climático considerado peligroso}

El "punto de inflexión" hace referencia a un límite concreto de incremento de las temperaturas atribuibles al hombre que, en caso de ser superado, desencadenaría una serie de crisis ambientales, económicas y sociales con serías consecuencias para el género humano y los ecosistemas ${ }^{10}$. En otras palabras, se trata del nivel de riesgo aceptable o el umbral de peligro de las interferencias humanas en el sistema del clima. Lógicamente, el grado de riesgo aceptable puede variar dependiendo de quién realice el juicio de valor, si se hace desde un punto de vista social o político, y en atención con el bien (ecosistemas, desarrollo, etc.) que se desee proteger. Las dificultades para determinar un punto preciso de inflexión se ven exacerbadas por el hecho de que la ciencia aún no tiene los medios tecnológicos que permitan realizar estimaciones del cambio climático sin margen de error.

En cualquier caso, desde un punto de vista del desarrollo, existe consenso entre los principales expertos en este campo en que se alcanzaría el punto de inflexión si el incremento en la temperatura promedio terrestre es superior a $\operatorname{los} 2^{\circ} \mathrm{C}$ en relación con

\footnotetext{
${ }^{7}$ IPCC, Cambio Climático 2007... cit., p. 36.

${ }^{8}$ Ibid.

${ }^{9}$ Op. cit., p. 44.

${ }^{10}$ El Artículo 2 de la CMNUCC habla de "interferencias peligrosas en el sistema climático"
} 
los niveles preindustriales ${ }^{11}$. Para evitar un aumento de las temperaturas por encima del punto de inflexión es necesario adoptar medidas de mitigación ${ }^{12}$ de GEI que permitan estabilizar su concentración en la atmósfera. Estas medidas deben adoptarse en un corto plazo ya que, como ha reconocido el Secretario General de las Naciones Unidas, Ban $\mathrm{Ki}-\mathrm{Moon}$, la comunidad internacional tiene menos de 10 años para reaccionar si se desean las consecuencias más peligrosas asociadas al cambio climático ${ }^{13}$.

\section{La estabilización de emisiones de GEI: Mitigación}

Los estudios del IPCC concluyen que, sin un cambio en los patrones de emisión actuales de GEI, el umbral de $2^{\circ} \mathrm{C}$ será superado con facilidad. Por ejemplo, estudios recientes indican que, en comparación con el año 2000, las emisiones de $\mathrm{CO}^{2}$ por el uso de energía aumentarán entre un $40 \%$ y $110 \%$ para el año $2030^{14}$. Por su parte, el Programa de las Naciones Unidas sobre el Desarrollo (PNUD) ha observado que, para tener un $50 \%$ de posibilidades de evitar superar el punto de inflexión en el cambio climático, se requiere estabilizar los GEI en concentraciones cercanas a las 450 partes por millón (ppm) de $\mathrm{CO}^{2} \mathrm{e}^{15}$, y ha advertido que si se estabilizan en $550 \mathrm{ppm}$ de $\mathrm{CO}^{2} \mathrm{e}$ aumentaría la probabilidad de traspasar el umbral a $80 \%{ }^{16}$.

11 BANCO MUNDIAL, Informe sobre el desarrollo mundial 2010: Desarrollo y cambio climático, Banco Internacional de Reconstrucción y Fomento/Banco Mundial, Washington, DC, 2010, p. 3; PNUD, Informe sobre Desarrollo Humano 2007-2008... cit., p. 3.

${ }^{12}$ Se entiende por "mitigación" la intervención humana para reducir las fuentes y mejorar el efecto de los sumideros de GEI. IPCC, Climate Change 2001: Impacts, Adaptation, and Vulnerability, Cambridge University Press, Cambridge, 2001, p. 89.

${ }^{13}$ UN News Centre, “Opening remarks..." cit.

${ }^{14}$ IPCC, "Summary for Policymakers", Metz, B., Davidson, O.R., Bosch, P.R., Dave, R., Meyer, L.A., (eds,), Climate Change 2007: Mitigation. Contribution of Working Group III to the Fourth Assessment Report of the Intergovernmental Panel on Climate Change, Cambridge University Press, CambridgeNueva York, 2007, p. 4.

${ }^{15} \mathrm{El}$ contenido de $\mathrm{CO}^{2}$ acumulado en la atmósfera se expresa en partes por millón (ppm) en un volumen determinado de aire. Ahora bien, en virtud de que además del $\mathrm{CO}^{2}$ existen otros gases que crean el efecto invernadero, con frecuencia se hace referencia a otro tipo de mediciones que los incluyen y se expresan en $\mathrm{CO}^{2}$ e. Este tipo de mediciones toman en cuenta cuánto calor puede retener un grupo de gases en relación con la cantidad de $\mathrm{CO}^{2}$ que sería necesaria para producir una retención de calor equivalente, las dos cantidades se suman y el $\mathrm{CO}^{2}$ e es el resultado. La suma de otros gases aumenta la concentración del

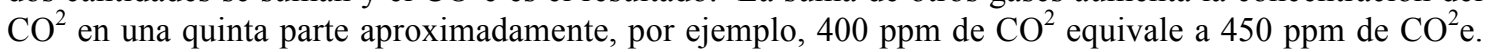
ADAM, D., "Explainer: Global carbon reduction targets", The Guardian, 7 Octubre 2008. Recuperado el 10 de marzo de 2010 de: http://www.guardian.co.uk/environment/2008/jun/10/carbonemissions

${ }^{16}$ PNUD, Informe sobre Desarrollo Humano 2007-2008 ... cit., pp. 6-7. 
En ese sentido, es interesante observar que a principios de la era industrial había 280 ppm de $\mathrm{CO}^{2} 17$ y actualmente hay 389,91 ppm (la más alta en los últimos 65,000 años), lo que equivale a $440 \mathrm{ppm}$ de $\mathrm{CO}^{2}$ e, con una tasa de crecimiento anual de 2 ppm. ${ }^{18} \mathrm{Si}$ continuamos con las tendencias actuales, se estima una posible estabilización para el siglo XXI en más de $750 \mathrm{ppm}$ de $\mathrm{CO}^{2}$ e con un aumento potencial en la temperatura de $5^{\circ} \mathrm{C}^{19}$. El Banco Mundial advierte que en un supuesto de esta naturaleza afectaría profundamente las opciones de desarrollo mundiales, especialmente en los países más pobres $^{20}$. Para evitar que las temperaturas suban más de $2^{\circ} \mathrm{C}$, las emisiones globales de GEI deberían alcanzar su pico en 2015 de ahí disminuir progresivamente un 40-45\% para el año 2050 en comparación con los niveles de $1990^{21}$.

\section{EL CAMBIO CLIMÁTICO Y SUS VÍNCULOS CON EL DESARROLLO}

La comunidad internacional tiene importantes retos en materia de desarrollo. Por ejemplo, en el 2005, el número de personas que vivía con menos de $\$ 1.25$ dólares al día era de 1,400 millones; a esta cifra en el 2009 se sumaron de 55 millones a 90 millones de personas más. Adicionalmente, los esfuerzos de erradicación de la pobreza están viendo revertir sus logros debido al alto precio de los alimentos. Más aún, la hambruna en los países en desarrollo está al alza, de ser el $16 \%$ en 2006 ha pasado a ser el 17\% en 2008. Los niños, como sucede a menudo, son el grupo más afectado: un cuarto de los niños en las regiones en desarrollo sufren de bajo peso $^{22}$ y tras la crisis las perspectivas de futuro no son muy esperanzadoras en algunas de las zonas más necesitadas ${ }^{23}$.

En este ámbito del desarrollo, los denominados objetivos del Milenio representan la principal aspiración y esperanza de la comunidad internacional. El 8 septiembre del año

\footnotetext{
${ }^{17}$ IPCC, Cambio climático 2007... cit., p. 37.

${ }^{18}$ Earth System Research Laboratory, National Oceanic \& Atmospheric Administration (U.S. Department of Commerce), Trends in Atmospheric Carbon Dioxide. Recuperado el 10 de marzo de 2010 de: http://www.esrl.noaa.gov/gmd/ccgg/trends/\#mlo

${ }^{19}$ PNUD, Informe sobre Desarrollo Humano 2007-2008 ... cit., p. 7.

${ }^{20}$ BANCO MUNDIAL, Informe el desarrollo mundial 2010... cit., p. 10.

${ }^{21}$ BAUMERT, K. A. et. al., Navigating the Numbers: Greenhouse Gas Data and International Climate Policy, World Resources Institute, p. 2.

${ }^{22}$ UN et. al., The Millennium Development Goals Report 2009, United Nations Department of Economic and Social Affairs (DESA), Nueva York, 2009, p. 4.

${ }^{23}$ FMI, Perspectivas de la economía mundial 09: Sustentar la recuperación, International Monetary Fund, Publication Services, Washington, DC, 2009, p. 54.
} 
2000, la Asamblea General de las Naciones Unidas adoptó la Declaración del Milenio en la que se contiene el compromiso político de 189 países de hacer frente algunas de las manifestaciones más graves y deshumanizadoras del subdesarrollo, fijándose como fecha límite el año 2015 para lograr objetivos concretos en materia de desnutrición, erradicación de la pobreza, sanidad, falta de vivienda, exclusión, desigualdad, educación, sostenibilidad ambiental ${ }^{24}$. El cambio climático es considerado por las principales organizaciones internacionales como uno de los mayores obstáculos para lograr cumplir los objetivos del mileno ${ }^{25}$.

\section{Los efectos del cambio climático en el desarrollo}

El incremento en las temperaturas provocará perdidas económicas en todo el mundo, pero principalmente a los países más pobres, ya que afectarán a los factores de producción desde las infraestructuras hasta las capacidades humanas. ${ }^{26}$ Lógicamente, mientras mayor sea incremento en las temperaturas más cuantiosas serán las pérdidas.

La seguridad alimentaria, la disponibilidad de agua, el equilibrio ecológico, la salud y la estabilidad social son algunos de los aspectos relacionados con el desarrollo que sufrirán negativamente los efectos del cambio climático. Todos estos factores de forma conjunta incidirán en las opciones de desarrollo, especialmente de los países más pobres. A continuación, sin ánimo de ser exhaustivos, mencionares algunos de los impactos del cambio climático en algunos sectores de importancia para el desarrollo.

\subsection{El declive en la producción agrícola y la seguridad alimentaria}

Según datos de la FAO, actualmente existen en el mundo aproximadamente 1.020 millones de personas que padecen desnutrición -el mayor número desde 1970-. ${ }^{27}$ Los retos en términos de seguridad alimentaria son importantes toda vez que para satisfacer la demanda de alimentos será necesario duplicar la producción para el año $2030{ }^{28}$ En

24 ASAMBLEA GENERAL DE LAS NACIONES UNIDAS, Resolución A/RES/55/2*, de 13 de septiembre de 2000 .

${ }^{25}$ UN, The Millennium Development Goals Report 2009... cit., p. 5.

${ }^{26}$ ELIZALDE, M. A., Sustainable Development in the Context of Climate Change: Overriding Priority of the South, South Centre Analytical Note SC/GGDP/AN/ENV6, Ginebra, 2008, p. 3.

${ }^{27}$ FAO, The State of Food Insecurity in the World 2009: Impacts and Lessons Learned, FAO, Roma, 2009, p. 11.

${ }^{28}$ FAO, The State of Food Insecurity in the World 2006: Eradicating World Hunger, FAO, Roma, 2006, pp. 5-6. 
este punto, la producción agrícola, una actividad altamente sensible a los factores climáticos, juega un papel muy importante ${ }^{29}$. Más aún, la relevancia de la producción agrícola para el desarrollo resulta evidente si consideramos que dos tercios de los pobres del planeta habitan en zonas rurales ${ }^{30}$.

En cuanto a los efectos del cambio climático en este ámbito, se estima que el incremento de las temperaturas hará cambiar los patrones de lluvia afectando la disponibilidad de agua para los riegos. La sequía afectará entre 60 y 90 millones de hectáreas más y las tierras cuyo riego depende de las lluvias de temporal sufrirán pérdidas de 26.000 millones de dólares ${ }^{31}$ de nuestros días al año $2060^{32}$. En África, las zonas secas serán más secas y las húmedas más húmedas, con proyecciones de perdidas de productividad agrícola de un $16 \%$ para el año $2080^{33}$. Otras zonas en desarrollo que se verán afectadas por la pérdida de productividad son América Latina y Asia Meridional, agudizando los problemas de pobreza rural $^{34}$. Con la perdida en la productividad agrícola caerá la disponibilidad de alimentos exacerbando aún más la inseguridad alimentaria que ya impera en algunos países ${ }^{35}$.

\subsection{La disponibilidad de agua}

Estudios del PNUD indican que la escasez de agua, alta o moderada, afecta actualmente a un tercio de la población mundial ${ }^{36}$. Como ya hemos comentado, el calentamiento global incidirá en los patrones de lluvias lo que se traducirá en un incremento en la frecuencia y severidad tanto de las inundaciones como de las sequías. En general, las zonas vulnerables a las inundaciones recibirán más agua, mientras que las que son

\footnotetext{
${ }^{29}$ Véase en general FAO, The State of Food Insecurity in the World 2009 ... cit.

30 WORLD BANK, Global Economic Prospects 2006: Economic Implications of Remittances and Migration, World Bank, Washington, DC, 2006, p. 10.

${ }^{31}$ En esta nota las referencias a los "dólares" debe entenderse hechas a los dólares de los Estados Unidos de América.

${ }^{32}$ PNUD, Informe sobre Desarrollo Humano 2007-2008 ... cit., p. 9.

${ }^{33}$ BANCO MUNDIAL, Informe el desarrollo mundial 2010... cit., p. 6.

${ }^{34}$ PNUD, Informe sobre Desarrollo Humano 2007-2008 ... cit., p. 9.

${ }^{35}$ FAO, The State of Food Insecurity in the World 2009... cit., p. 11.

${ }^{36}$ UNDP, Human Development Report 2006: Beyond Scarcity: Power, Poverty and the Global Water Crisis, Palgrave MacMillan, Nueva York, 2006, p. 15.
} 
propensas a las sequías recibirán menos ${ }^{37}$. La subida del nivel del mar, por otra parte, puede producir infiltraciones de agua salada en ríos de agua dulce, limitando la disponibilidad de agua para el consumo humano ${ }^{38}$.

\subsection{Los efectos en el medio ambiente}

El clima es uno de los factores básicos que determinan el funcionamiento de los ecosistemas. De este modo, el cambio climático representa gran peligro para el equilibrio ecológico. El calentamiento de los mares está afectando a los arrecifes de coral, muy sensibles a las altas temperaturas e importantes para la cadena alimenticia. Los patrones de migración de diversas especies empiezan a cambiar, afectando la nidificación y la disposición de alimento para las crías. Además, los expertos aseguran que un incremento de las temperaturas de $3^{\circ} \mathrm{C}$ podría implicar la extinción de entre $20 \%$ y $30 \%$ de las especies terrestres, ya que no todas podrán adaptarse ${ }^{39}$.

\subsection{Los efectos en la salud humana}

El impacto del cambo climático en la seguridad alimentaria, en la disponibilidad de agua, en los ecosistemas afecta a su vez a la salud humana. Las crisis ambientales, que actualmente ya se cobran la vida de muchas personas alrededor del mundo, se verán agudizadas convirtiéndolas en un peligro mayor. Pero además, existen otras amenazas para la salud asociadas al cambio climático. Por ejemplo, se estima que las principales epidemias mortales podrían llegar a zonas que antes se encontraban libres de ellas. Este es el caso del dengue, actualmente se encuentra en altitudes superiores a las comunes; esto implica una ampliación del ámbito geográfico potencial de la enfermedad. En cifras, se estima que el paludismo podría afectar a 220 a 400 millones de personas ${ }^{40}$.

\footnotetext{
${ }^{37}$ ELIZALDE, M. A., "Una perspectiva amplia del cambio climático desde el Derecho internacional público", Institut de Drets Humans de Catalunya, Derechos humanos y cambio climático, Institut de Drets Humans de Catalunya, Barcelona, 2008, p. 42.

${ }^{38}$ UNDP, Human Development Report 2006 ... cit., p. 15.

${ }^{39}$ PNUD, Informe sobre Desarrollo Humano 2007-2008... cit., p. 10.

${ }^{40}$ Ibid.
} 


\subsection{Los efectos en la estabilidad social}

Las tensiones entre grupos humanos pueden surgir conforme los impactos en el desarrollo se vayan haciendo más evidentes, afectando la estabilidad social. Por ejemplo, un estudio las Naciones Unidas señala al cambio climático, entre otros factores, como una de las fuentes del conflicto de Darfur, en Sudán, que ha causado aproximadamente 500,000 muertes ${ }^{41}$. Más aún, como ha observado el IPCC, existe un serio riesgo de que la falta de oportunidades de desarrollo asociadas al cambio climático favorezca migraciones masivas de personas ${ }^{42}$.

\section{La dimensión Norte-Sur}

El reto del cambio climático presenta ángulos o dimensiones distintas según se aborde desde la perspectiva de los países desarrollados, también denominados del Norte, o de los países en vías de desarrollo, también conocidos como países del Sur. Entre unos y otros existen importantes diferencias en relación con el grado de vulnerabilidad frente al cambio climático, su responsabilidad histórica y su capacidad de adaptación.

\subsection{La diferencia en la vulnerabilidad frente al cambio climático}

Aunque el calentamiento global es una amenaza que se cierne sobre toda la comunidad internacional, los países en desarrollo, al ser más vulnerables, sufrirán los efectos más severos. Las cifras ilustran claramente la gran diferencia de vulnerabilidad frente a las crisis climáticas que existe entre los países desarrollados y los países en vías de desarrollo. Cada año comprendido entre el 2000 y el 2004 los desastres medioambientales afectaron a más de 260 millones de personas, el 2\% de éstas vivían en países desarrollados y el 98\% restante en países en vías de desarrollo. Puesto de una manera diferente, uno de cada 1,500 habitantes de los países de la OCDE fue víctima de fenómenos climáticos; mientras que la proporción en los países en vías de desarrollo fue de una por cada $19^{43}$. Un ejemplo en términos económicos lo aporta el Banco Mundial, estima que un incremento en la temperatura de $2^{\circ} \mathrm{C}$ podría provocar perdidas del $4 \%$ al

\footnotetext{
${ }^{41}$ UNEP, Sudan: Post-Conflict Environmental Assessment, PNUMA, Nairobi, 2007, p. 15.

${ }^{42}$ IPCC, Climate Change $2001 \ldots$ cit., p. 454.

${ }^{43}$ PNUD, Informe sobre Desarrollo Humano 2007-2008 ... cit., p. 8.
} 
5\% del ingreso anual per capita en África y Asia, mientras que las pérdidas serían mínimas para los países desarrollados ${ }^{44}$.

La mayor vulnerabilidad al cambio climático de los países en desarrollo es producto de una combinación de factores socio-económicos y geográficos ${ }^{45}$. Por lo que toca a los factores socioeconómicos, y a diferencia de lo que sucede en los países desarrollados, los países en vías de desarrollo carecen de recursos tecnológicos, humanos y financieros, así como de las infraestructuras e instituciones necesarias para reducir y gestionar los riesgos asociados con los cambios climáticos. En cuanto a los factores geográficos, un importante número de países en desarrollo están ubicados en zonas tropicales o subtropicales proclives a variaciones climáticas extremas que se verán exacerbadas por el cambio climático; mientras que otros se ubican en latitudes bajas, en zonas costeras o son pequeñas islas lo que los hace particularmente vulnerables a la subida del nivel del mar ${ }^{46}$.

\subsection{La responsabilidad común pero diferenciada}

Los países en desarrollo perciben la amenaza del cambio climático como una gran injusticia y no les falta razón. El cambio climático de origen antropogénico puede atribuirse a los países desarrollados en cuyos procesos de industrialización y crecimiento económico han emitido el 75\% del total histórico de GEI. Todavía hoy, los países desarrollados, que representan sólo el $15 \%$ de la población mundial, son responsables de la mitad de las emisiones de GEI aproximadamente ${ }^{47}$. En contraste, sin contar a los grandes países en vías de desarrollo como China, la India y Brasil, cerca de más de 100 países en vías de desarrollo no superan conjuntamente el 10\% de las emisiones de GEI mundiales ${ }^{48}$. Actualmente, China es, después de los Estados Unidos (con el $21 \%$ de las emisiones globales), la segunda mayor fuente de emisiones agregadas de GEI a nivel mundial (15\% aproximadamente); aunque si se consideran las emisiones per capita, sigue estando muy por debajo de los niveles de los países desarrollados, aproximadamente una sexta parte de las emisiones de un ciudadano en

\footnotetext{
${ }^{44}$ BANCO MUNDIAL, Informe el desarrollo mundial 2010... cit., p. 5.

${ }^{45}$ Op. cit., p. 6.

${ }^{46}$ ELIZALDE, Sustainable Development in the Context of Climate Change... cit., p. 5.

${ }^{47}$ PNUD, Informe sobre Desarrollo Humano 2007-2008 ... cit., p. 7.

${ }^{48}$ BAUMERT, Navigating the Numbers... cit., pp. 12 y 32.
} 
Estados Unidos ${ }^{49}$. Por lo demás, se espera que el mayor aumento de emisiones en el futuro tenga lugar en los países en vías de desarrollo.

La diferencia en las responsabilidades históricas y de vulnerabilidad en el contexto del cambio climático entre países desarrollados y países en vías de desarrollo ha dado lugar al nacimiento del principio de responsabilidades comunes pero diferenciadas ${ }^{50}$. Conforme a este principio, a los países desarrollados les corresponde la responsabilidad primordial de mitigar el cambio climático, por un lado, y de ayudar a los países en vías de desarrollo a adaptarse, por otro. La diferencia entre mitigación y adaptación es que la primera está destinada a reducir las emisiones de gases GEI, mientras que la adaptación se refiere a las medidas necesarias para evitar o reducir el impacto del cambio climático en un país determinado. Aquí conviene enfatizar que ambas son indispensables en la lucha contra el cambio climático.

\subsection{El imperativo de la adaptación}

La aplicación de una estrategia ambiciosa de mitigación es necesaria para evitar que el cambio climático no supere el umbral o punto de inflexión a partir del cual tendrían lugar las consecuencias más peligrosas. Sin embargo, en virtud de que los GEI una vez emitidos quedan atrapados en la atmósfera por muchos años, los beneficios de una estrategia exigente y exitosa de mitigación se empezarían a sentir sólo después de 20 años. Más concretamente, los expertos observan que no es posible evitar que, al menos en las siguientes dos décadas, el cambio climático haga sentir parte de sus consecuencias $^{51}$. De este modo, la adaptación deja de ser una opción para convertirse en un imperativo, especialmente para los países en vías de desarrollo.

En los últimos años, diversos estudios han intentado elaborar estimaciones de los costes necesarios para lograr la adaptación. Una de las primeras conclusiones es que el coste de la adaptación se incrementará conforme más aumenten las temperaturas. Por lo tanto, actuar a tiempo en el ámbito de la mitigación implica a su vez un coste más bajo de

\footnotetext{
${ }^{49}$ VANDENBERG, M. P., "Climate Change: The China Problem”, Southern California Law Review, Vol. 81, 2008, p. 917.

${ }^{50}$ Artículo 4.1 de la CMNUCC.

${ }^{51}$ IPCC, "Informe del Grupo de Trabajo III - Mitigación del Cambio Climático, Pasado, presente y futuro: tendencias de emisión”. Recuperado el 12 de marzo de 2010 de http://www.ipcc.ch/publications_and_data/ar4/wg3/es/tssts-1-2.html
} 
adaptación. Aunque los estudios presentan diferencias y no son siempre directamente comparables, en términos generales, indican que el coste de adaptación para los países en vías de desarrollo es de 30-90 mil millones de dólares anuales ${ }^{52}$. No obstante, es importante decir que está suma no incluye todas las posibles variantes, por lo tanto, sirve simplemente de referencia.

Ahora bien, consideraciones elementales de justicia indican que los países desarrollados, al ser responsables históricos del cambio climático, tienen el deber de ayudar económicamente a los países en vías de desarrollo a adaptarse. Como ya hemos indicado antes, los recursos económicos determinan en gran medida la capacidad de adaptación. Para ilustrar este punto sirva de ejemplo una comparación entre los Países Bajos y Bangladesh. Estos países, dada su ubicación geográfica, son muy vulnerables al incremento del nivel del mar, si bien, su situación es muy distinta si se ve desde una perspectiva económica y de capacidad para adaptarse. Los Países Bajos tienen previsto destinar \$100 dólares por cada uno de sus habitantes hasta el año 2100, una cifra imposible de igualar por el segundo cuyo ingreso anual per capita es de 450 dólares ${ }^{53}$.

Las diferencias en su responsabilidad histórica, de vulnerabilidad y de capacidad de adaptación son las variantes que están presentes en las negociaciones internacionales que buscan establecer el régimen internacional de lucha contra el cambio climático que permita a la humanidad no superar el punto de inflexión.

\section{EL CAMINO HACIA EL NUEVO RÉGIMEN DEL CAMBIO CLIMÁTICO}

Las opciones en materia de desarrollo de las nuevas y futuras generaciones dependen en gran medida de que la Comunidad internacional y sus dirigentes actuales logren un acuerdo internacional ambicioso en objetivos de mitigación y adaptación que suceda al régimen internacional existente.

\footnotetext{
${ }^{52}$ UNFCCC, Potential costs and benefits of adaptation options: A review of existing literature (Technical paper), UNFCCC Doc. FCCC/TP/2009/2, 7 de diciembre de 2009, p. 5.

${ }^{53}$ BANCO MUNDIAL, Informe el desarrollo mundial 2010... cit., p. 7.
} 


\section{El régimen internacional en vigor}

La Convención Marco de las Naciones Unidas sobre el Cambio Climático (CMNUCC) y el Protocolo de Kioto constituyen actualmente las únicas respuestas normativas vinculantes multilateralmente acordadas para hacer frente al reto que representa el calentamiento global ${ }^{54}$. La CMNUCC define los objetivos y compromisos en términos generales, mientras que el Protocolo de Kioto contiene obligaciones más concretas.

El objetivo de la CMNUCC es lograr la estabilización de las concentraciones de GEI en la atmósfera de modo que se eviten las interferencias humanas peligrosas en el sistema del clima ${ }^{55}$. Además del principio de responsabilidades comunes pero diferencias, la CMNUCC reconoce las necesidades especiales de los países en desarrollo, la responsabilidad principal de los países desarrollados de adoptar medidas de mitigación y de ayudar económicamente a los países en desarrollo a adaptarse ${ }^{56}$.

El Protocolo de Kioto a la CMNUCC establece los compromisos específicos de reducción de emisiones en su Anexo B. Los países que figuran en el Anexo I en la CMNUCC - 37 países desarrollados y la CE - deben reducir sus emisiones en no menos $5 \%$ en relación con a los nieves existentes en 1990 para el periodo comprendido entre el 2008 y $2012^{57}$. Con independencia de que los compromisos de reducción de emisiones no son suficientes para evitar el cambio climático peligroso, la eficacia del Protocolo de Kioto se ve seriamente afectada por el hecho de que los dos principales emisores de GEI en el mundo, Estados Unidos y China, no tienen compromisos de reducción; el primero por no estar vinculado al acuerdo y el segundo por no figurar entre los países Anexo I de la CMNUCC. Más aún, estudios del PNUD indican que no todos los países con compromisos de reducción vinculantes están en el camino de cumplirlos ${ }^{58}$.

Así, es evidente que si se desea evitar hipotecar el futuro de las próximas generaciones, la comunidad internacional debe encontrar el camino que permita el establecimiento de un régimen internacional post-Kioto para regir en este ámbito a partir del 2012 y que

\footnotetext{
${ }^{54}$ Véase el Protocolo de Kioto a la Convención Marco de las Naciones Unidas sobre el Cambio Climático, hecha en Kioto, Japón, el 11 de diciembre de 1997 (BOE, de 8 de febrero de 2005).

${ }^{55}$ Artículo 2 CMNUCC.

${ }^{56}$ Artículos 4.1, 3.2, 3.1, y 4.4 CMNUCC, respectivamente.

${ }^{57}$ Artículo 3.1 del Protocolo de Kioto.

${ }^{58}$ PNUD, Informe sobre Desarrollo Humano 2007-2008... cit., p. 7.
} 
sea suficientemente eficaz en evitar un incremento de las temperaturas de $2^{\circ} \mathrm{C}$ por arriba de los niveles preindustriales.

\section{Los avances en las negociaciones de un régimen post-Kioto}

En septiembre de 2009, en Copenhague, tuvo lugar la $15^{\mathrm{a}}$ reunión de la Conferencia de la Partes de la CMNUCC de la que se esperaba que los líderes mundiales lograran, por un lado, un acuerdo multilateral vinculante que constituyera el establecimiento del régimen post-Kioto con compromisos de reducción definidos y, por otro, un fondo para ayudar a los países en vías de desarrollo a adaptarse al cambio climático ${ }^{59}$. No obstante, la Conferencia fue una decepción ya que no se lograron ninguno de los dos objetivos.

Las divergencias que han provocado el colapso de las negociaciones implican en términos generales cuestiones de desarrollo. Existen diferentes frentes, pero los polos principales enfrentados en las negociaciones están representados por los Estados Unidos y China que se culpan uno a otro de haber obstaculizado el proceso de negociación ${ }^{60}$. El primero de estos países, preocupado por consideraciones de competencia económica, ha sido reticente a adoptar compromisos de reducción de emisiones vinculantes si los países en vías de desarrollo no hacen lo mismo. Esta posición ha sido sostenida desde 1992, fecha en que se adoptó la CMNUCC, hasta Copenhague en 2009, pasando por Kioto, en donde el senado de los Estados Unidos se negó a conceder la autorización para ratificar el Protocolo en $1997^{61}$. El argumento principal es que las proyecciones a mediano plazo indican que el mayor aumento de emisiones de GEI globales tendrá lugar en los países en vías de desarrollo ${ }^{62}$. China, por su parte, refleja la posición de los países en desarrollo, se niega asumir compromisos obligatorios de reducción de emisiones que ralenticen su crecimiento económico y sostiene que las reducciones obligatorias y los fondos para la adaptación deben provenir de los países desarrollados, al ser éstos los

\footnotetext{
${ }^{59}$ WILEY, L. F., "Healthy Planet, Healthy People: International Global Health into the International Response to Climate Change", Journal of Environmental Law and Litigation, Vol. 24, 2009, pp. 204-205.

${ }^{60}$ GONZALEZ, Carmen G.; "China in Latin America: Law, Economics, and Sustainable Development", Environmental Law Reporter News \& Analysis, Vol. 40, febrero 2010, p. 10182.

${ }^{61}$ Véase 105th Congress, 1st. Session, S. Res. 98, June 12, 1997, donde se expresa la opinión del Senado en relación con las condiciones para que Estados Unidos firme cualquier tipo de acuerdo internacional sobre emisiones de gases de efecto invernadero bajo la Convención Marco de las Naciones Unidas sobre Cambio Climático.

${ }^{62}$ Ibid.
} 
responsables históricos de causar el cambio climático ${ }^{63}$. Aunque China ha realizado declaraciones indicando que tiene intensión de reducir sus emisiones entre un $40 \%$ a $45 \%$ para 2020 en relación con las emisiones de 2005, se niega a aceptar un control internacional sobre su cumplimiento ${ }^{64}$.

En las últimas horas de la Conferencia de Copenhague, Estados Unidos y China dieron la falsa impresión de acercamiento. Un grupo liderado por Estados Unidos, que al final terminó por convencer e incluir a China y a otros grandes países en desarrollo, adoptó una declaración política no vinculante denominada "el Acuerdo de Copenhague" 65 . Conviene aclarar que esta declaración, además de no constituir ningún cambio al régimen existente, no forma parte de los resultados de la Conferencia - aunque en ésta se "tomó nota de ella" - toda vez que fue negociada al margen de las de la conferencia principal y sólo entre algunos países participantes.

Lo que apoyan el acuerdo de Copenhague enfatizan que éste contiene acuerdos de transferir billones de dólares para ayudar a los países en desarrollo a adaptarse y que representa un primer paso importante hacia la adopción de un acuerdo obligatorio, equitativo y ambicioso ${ }^{66}$. Sin embargo, los representantes de los países en desarrollo más vulnerables al cambio climático (incluidas las naciones africanas y pequeñas islas Estado) han denunciado con severidad que el Acuerdo de Copenhague no contenga reducciones específicas y que se hubiera excluido al G77 del proceso de negociación lo que llevo a que se le calificara de un coup d'etat en contra de la ONU_ ${ }^{67}$. En efecto, en el acuerdo de Copenhague los países participantes simplemente reconocen que es necesario adoptar las medidas de mitigación para evitar que el calentamiento global sea superior a $2^{\circ} \mathrm{C}$ en relación con los niveles preindustriales ${ }^{68}$. Sin embargo, no establece

\footnotetext{
63 ZONGLAI, W., BIN, H., "Development and History: China's Reform and Opening-Up and International Law", Chinese Journal of International law, Vol. 9, marzo 2010, p. 193.

${ }^{64}$ MILLER, T., “Obama Urges Action, Meets with Chinese Premier”, PBS, 18 de diciembre de 2009. Recuperado el 2 de enero de 2010 de http://www.pbs.org/newshour/updates/environment/julydec09/copenhagen_12-18.html

${ }^{65}$ Report of the Conference of the Parties on its fifteenth session, held in Copenhagen from 7 to 19 December 2009. FCCC/CP/2009/11/Add.1, 30 de marzo de 2010, p. 5

${ }^{66}$ BURLESON, E., "Climate Change Consensus: Emerging International Law", William and Mary Environmental Law and Policy Review, Vol. 34, 2010, p. 551.

${ }^{67}$ Taipei Times. (2009, 20 de diciembre), “Copenhaguen Talks: Climate deal faces poor nations' fury",

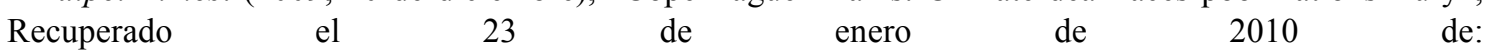
http://www.taipeitimes.com/News/world/archives/2009/12/20/2003461353

${ }^{68}$ Acuerdo de Copenhague, p. 5.
} 
compromisos específicos y cuantificados de reducción ni fechas de cumplimiento, dejando abierto el principal frente de divergencia entre los dos grandes emisores de GEI en el mundo 69 . Por otro lado, la declaración el "Acuerdo de Copenhague" también contiene un compromiso político por parte de los países desarrollados de movilizar $\$ 30$ mil millones de dólares anuales de 2010 a 2012 y \$100 mil millones de dólares anuales hasta 2020 en calidad de fondos de adaptación para ayudar a los países en vías de desarrollo, con preferencia a los más vulnerables ${ }^{70}$.

Sin embargo, en ausencia de medidas concretas encaminadas a la estabilización e incluso suponiendo que los fondos de adaptación fueran suficientes y bien utilizados, las medidas de adaptación sólo retardarían las consecuencias del cambio climático. Ian Fry, representante de Tuvalu, ilustra claramente el sentir de los países más vulnerables al cambio climático, al decir que el Acuerdo de Copenhague era equivalente a que se les ofreciera treinta piezas de plata a cambio de traicionar a su pueblo y su futuro ${ }^{71}$.

Es claro que la comunidad internacional deben encontrar la formula para lograr una cooperación multilateral efectiva en la lucha contra el cambio climático.

\section{Los principales retos a superar en el camino hacia un régimen post-Kioto}

Muchos son los retos que la comunidad internacional ha de resolver en el camino hacia el establecimiento de un marco normativo que suceda al actual. A continuación mencionaremos brevemente algunos de los más importantes.

\subsection{La cooperación multilateral efectiva}

El cambio climático es un reto global y todos los países deben aportar esfuerzos y voluntad para hacerle frente. Por lo tanto, lograr la cooperación de todos los países para luchar contra el cambio climático con base en el principio de responsabilidades comunes pero diferenciadas es, sin duda, el principal reto que enfrenta la comunidad

\footnotetext{
${ }^{69}$ Véase, por ejemplo, la Carta del Primer Ministro de la República Popular China dirigida al Primer Ministro Danés en relación con el acuerdo de Copenhague en la que insiste en que las reducciones de emisiones de los países en desarrollo no deben ser vinculantes, sino voluntarias. UNFCCC, Communications received from Parties in relation to the listing in the chapeau of the Copenhagen Accord. Recuperado el 2/04/2010 de http://unfccc.int/meetings/items/5276.php

${ }^{70}$ Report of the Conference... cit., p. 6.

71 “Copenhaguen Talks: Climate deal faces poor nations' fury” cit.
} 
internacional en este ámbito. Lógicamente, no todos los países tienen la misma capacidad o responsabilidad, sin embargo, ningún esfuerzo internacional será suficiente si los principales emisores de GEI no adoptan medidas para mitigar el calentamiento global. En particular, China y los Estados Unidos deben mostrar mayor voluntad de cooperación. No es lógico, por ejemplo, que los Estados Unidos, el mayor emisor de GEI, ofrezca reducciones del 4\% en relación con los niveles de 1990, como hizo al presentarse a negociar en la Conferencia de Copenhague el mes de Septiembre pasado ${ }^{72}$. China, por su parte, debe adoptar compromisos de reducción, si bien, en atención a sus necesidades de desarrollo, debe recibir ayuda internacional en forma de fondos para la adaptación y en transferencia de tecnología.

\subsection{La financiación}

En los últimos años se han adoptado compromisos políticos importantes en materia de ayuda financiera para el desarrollo y para la adaptación. De hecho, en el ámbito de la ayuda al desarrollo, en el año 2008 se alcanzaron niveles record; no obstante, existen compromisos aún no cubiertos como los 35 mil millones de dólares anuales anunciados por el Grupo de los Ocho en Gleneagles y los 20 mil millones de dólares anuales de ayuda para África ${ }^{73}$. Un problema adicional es que la recesión que han experimentado los países de la OCDE no sólo dificulta el cumplimiento de esos compromisos, sino que incluso puede generar una disminución de la ayuda al desarrollo cuyos montos se expresan en porcentajes de los ingresos nacionales de los donantes ${ }^{74}$.

Por esta razón, es importante que los fondos de adaptación constituyan recursos adicionales reales a los que se conceden bajo la forma ayuda al desarrollo. En otras palabras, el cumplimiento de los compromisos reflejados en el Acuerdo de Copenhague en relación con los fondos para la adaptación no debe llevar a una disminución en los recursos para la ayuda al desarrollo. El cambio climático es un problema adicional a los problemas de desarrollo que ya tienen muchos países, por lo tanto, la ayuda para la

\footnotetext{
${ }^{72}$ The Guardian. (2009, 19 de diciembre). "Low Targets, goals dropped: Copenhagen ends in failure". Recuperado 20 de marzo de 2010 de: http://www.guardian.co.uk/environment/2009/dec/18/copenhagendeal

${ }^{73}$ MDG Gap Task Force Report 2009 (2009, 16 de septiembre), "UN takes a stand on global aid, trade and debt commitments, as G20 prepares to meet". Recuperado el 10 de abril de 2010 de: http://www.un.org/millenniumgoals/

${ }^{74}$ UN et. al., The Millennium Development Goals Report 2009... cit., p. 4.
} 
adaptación debe ser complementaria a la ayuda al desarrollo. En este sentido, en un estudio reciente el FMI ha observado que el empleo, el crecimiento y la lucha contra la pobreza en los países en vías de desarrollo ha sufrido retrocesos importantes retrocesos en el último año, por lo tanto, la ayuda al desarrollo no debe interrumpirse, pues los avances duramente logrados hasta ahora correrían el riesgo de desvanecerse, especialmente en los países de África ${ }^{75}$.

\subsection{La transferencia de tecnología}

La capacidad de los distintos países para mitigar el cambio climático y adaptarse a sus efectos también depende del acceso a las tecnologías adecuadas ${ }^{76}$. Los países desarrollados normalmente están mejor equipados en términos de tecnología que los países en vías de desarrollo, ya que la innovación tecnológica depende de las inversiones en investigación y desarrollo $(\mathrm{I}+\mathrm{D})$. Un problema es que, aunque algunos países desarrollo cuentan con conocimientos tradicionales útiles en el ámbito de la adaptación, las tecnologías disponibles y utilizadas en los países desarrollados tanto para mitigar como para adaptarse no siempre están a su alcance. Más aún, si bien es verdad el régimen actual contiene normas que establecen compromisos en materia de transferencia de tecnología en sentido Norte-Sur, éstos no se están cumplimiento ${ }^{77}$.

Por lo tanto, el reto consiste en establecer un marco efectivo que ponga la tecnología a disposición de los que más la necesitan ${ }^{78}$. En particular, es importante evitar que el régimen internacional de la propiedad intelectual sea utilizado como un obstáculo para que tengan lugar las transferencias. En ese sentido, el uso de las licencias obligatorias puede ser un instrumento que permita encontrar un equilibrio entre la transferencia de tecnología y el régimen internacional de la propiedad intelectual. Adicionalmente, la transferencias de tecnología en sentido Sur-Sur, siempre que sea posible, debe contarse entre las opciones para mejorar la capacidad de mitigar y adaptación al cambio climático. Por último, conviene decir que, al parecer, las negociaciones internacionales

\footnotetext{
${ }^{75}$ FMI, Perspectivas de la economía mundial 09... cit., p. 54.

${ }^{76}$ IPCC 2001, Adaptation and Vulnerability... cit., p. 896.

${ }^{77}$ UNFCCC, Technologies for Adaptation to Climate Change, UNON Publishing Services, Bonn, 2006, p. 11.

${ }^{78}$ Artículo 4.5 de la CMNUCC.
} 
en materia de transferencia tecnológica en el contexto del cambio climático parecen estar más avanzadas y pronto puede haber un acuerdo ${ }^{79}$.

\section{CONCLUSIÓN}

El desarrollo económico de los países actualmente ricos e industrializados ha generado la amenaza del cambio climático que se cierne sobre toda la humanidad. Por otro lado, actualmente son temas relacionados con el desarrollo económico los que deben resolver los líderes mundiales para establecer un régimen internacional efectivo para luchar contra el cambio climático, evitando así un futuro incierto para las próximas generaciones. El principio de responsabilidades comunes pero diferencias de la CMNUCC es punto de partida, aunque muchos cambios de actitud son necesarios. Los países desarrollados deben adoptar las obligaciones principales de mitigación y deben aportar recursos financieros a los países en desarrollo para ayudarles a adaptarse. Los países del Sur tienen el derecho a desarrollarse para cubrir las necesidades de su población. No obstante, el crecimiento no debe ser a cualquier coste. En particular, los grandes países en vías de desarrollo, como China y la India, deben adoptar medidas de mitigación y en este esfuerzo deben recibir ayuda de la comunidad internacional, por ejemplo, en forma de recursos y transferencia de tecnología.

\section{BIBLIOGRAFÍA}

ADAM, D., "Explainer: Global carbon reduction targets", The Guardian, 7 octubre 2008. Recuperado el 10 de marzo 2010 de: http://www.guardian.co.uk/environment/2008/jun/10/carbonemissions

BANCO MUNDIAL, Informe sobre el desarrollo mundial 2010: Desarrollo y cambio climático, Banco Internacional de Reconstrucción y Fomento/Banco Mundial, Washington, DC, 2010.

BAUMERT, K. A. et. al., Navigating the Numbers: Greenhouse Gas Data and International Climate Policy, World Resources Institute.

\footnotetext{
${ }^{79}$ BURLESON, “Climate Change Consensus: Emerging International Law...” cit., p. 545.
} 
BURLESON, E., "Climate Change Consensus: Emerging International Law", William and Mary Environmental Law and Policy Review, Vol. 34, 2010, pp. 543-588.

ELIZALDE, M. A., Sustainable Development in the Context of Climate Change: Overriding Priority of the South, South Centre Analytical Note SC/GGDP/AN/ENV6, Ginebra, 2008.

— "Una perspectiva amplia del cambio climático desde el Derecho internacional público", Derechos humanos y cambio climático, Institut de Drets Humans de Catalunya, Barcelona, 2008, pp. 33-65.

FAO, The State of Food Insecurity in the World 2006: Eradicating World Hunger, FAO, Roma, 2006.

- The State of Food Insecurity in the World 2009: Impacts and Lessons Learned, FAO, Roma, 2009.

FMI, Perspectivas de la economía mundial 09: Sustentar la recuperación, International Monetary Fund, Publication Services, Washington, DC, 2009.

HUQ, S., REID, H., MURRAY, L. A., Climate change and development links, International Institute for Environment and Development, Londres, 2006.

IPCC, Climate Change 2001: Impacts, Adaptation, and Vulnerability, Cambridge University Press, Cambridge, 2001.

- Cambio climático 2007: Informe de sintesis. Contribución de los Grupos de trabajo I, II y III al Cuarto Informe de evaluación del Grupo Intergubernamental de Expertos sobre el Cambio Climático [Equipo de redacción principal: Pachauri R.K. y Reisinger, A. (directores de la publicación)], IPCC, Ginebra, Suiza, 2007.

— Summary for Policymakers, Metz, B., Davidson, O.R., Bosch, P.R., Dave, R., Meyer, L.A., (eds,), Climate Change 2007: Mitigation. Contribution of Working Group III to the Fourth Assessment Report of the Intergovernmental Panel on Climate Change, Cambridge University Press, Cambridge-Nueva York, 2007.

PNUD, Informe sobre Desarrollo Humano 2007-2008: La lucha contra el cambio climático: Solidaridad frente a un mundo dividido, PNUD, Nueva York, 2007.

UN et. al., The Millennium Development Goals Report 2009, United Nations Department of Economic and Social Affairs (DESA), Nueva York, 2009. 
UNDP, Human Development Report 2006: Beyond Scarcity: Power, Poverty and the Global Water Crisis, Palgrave MacMillan, Nueva York, 2006.

UNEP, Sudan: Post-Conflict Environmental Assessment, PNUMA, Nairobi, 2007.

UNFCCC, Technologies for Adaptation to Climate Change, UNON Publishing Services, Bonn, 2006.

VAnDEnBERG, M. P., "Climate Change: The China Problem”, Southern California Law Review, Vol. 81, 2008, pp. 905-958.

WILEY, L. F., "Healthy Planet, Healthy People: International Global Health into the International Response to Climate Change", Journal of Environmental Law and Litigation, Vol. 24, 2009, pp. 203-240.

WORLD BANK, Global Economic Prospects 2006: Economic Implications of Remittances and Migration, World Bank, Washington, DC, 2006.

ZONGLAI, W., BIN, H., "Development and History: China's Reform and Opening-Up and International Law”, Chinese Journal of International law, Vol. 9, marzo 2010. 